\title{
Relationship of women's reproductive health and menstrual problems with sleep and circadian rhythm
}

\author{
Yoko Komada ${ }^{1}$
}

Published online: 5 December 2019

(C) Japanese Society of Sleep Research 2019

Menses-related health problems are experienced by many women during their fertile years. These menstrual symptoms can significantly impact women's daily lives. Several studies have investigated sleep quality across the menstrual cycle. For example, individual sleep quality has been reported to be worse during the premenstrual phase and menstruation [1]. Women who experience severe premenstrual syndrome have reported significant decline in sleep quality, especially during the late luteal phase rather than with the early follicular phase [1]. Thus, women's sleep is commonly affected by menstrual cycles and menstrual problems.

A cross-sectional survey of college students published in Sleep and Biological Rhythms [2] has shown that the prevalence rate of premenstrual dysphoric disorder (PMDD), premenstrual syndrome (PMS), and no/mild PMS was $12.3 \%$, $20.6 \%$, and $67.1 \%$, respectively. Daytime sleepiness measured by the Japanese version of the Epworth Sleepiness Scale (JESS) was highest in female students with PMDD, followed by those with PMS, and lowest in those with no/mild PMS. It is interesting to note that women with no/mild PMS still experienced higher daytime sleepiness than male students [2]. The underlying mechanism for the difference in daytime sleepiness between men and women remains unclear. The authors suggested the serum concentration rise of progesterone in luteal phase could be causing daytime sleepiness, suggesting sleepiness in women in follicular phase would be comparable to that in men [2]. A study conducted among shiftwork nurses revealed that musculoskeletal pain was associated with both insomnia and daytime sleepiness [3]. The relationship between sleep problems and pain is bidirectional, potentially forming a vicious cycle. Women suffering from menstrual pain or chronic pain are more prone to lose control over their regular sleep, thereby affecting their daily life and general health.

Reproductive hormones not only regulate reproductive function during the menstrual cycle, but also influence sleep

Yoko Komada

komay@my-pharm.ac.jp

1 Liberal Arts, Meiji Pharmaceutical University, Tokyo, Japan and circadian rhythms [4]. According to a study of young adults, sleep disturbance as well as psychological distress in daily life are associated with menstrual problems. Women with sleep-related difficulties experienced significantly more severe menstrual symptoms and tended to have irregular or a relatively lengthy menstrual cycle than those without sleeprelated difficulties [5]. Studies investigating the relationship between sleep and menstruation have been limited and many aspects remain unclear due to methodological issues such as heterogeneous cycle lengths and a lack of ovulation timing measurements. Future researches should focus on the association between sleep, circadian rhythms, menstrual symptoms, menstrual cycle, and reproductive health. Since a large number of women suffer from menstrual symptoms and sleep problems, considerably impacting women's lives and health, it is imperative to conduct a detailed study. Thus, promoting studies in this field and increasing public awareness can improve women's quality of life and health significantly.

\section{References}

1. Baker FC. Sex differences and menstrual-related changes in sleep and circadian rhythms. Principles and practice of sleep medicine, 6th edn. pp.1516-24. https://www.amazon.com/Principles-Pract ice-Sleep-Medicine-Kryger/dp/032324288X

2. Miura J, Honma R. Daytime sleepiness in relation to gender and premenstrual symptoms in a sample of Japanese college students. Sleep Biol Rhythms. 2019. https://doi.org/10.1007/s41105-019-00236-x

3. Katsifaraki M, Nilsen KB, Wærsted M, Knardahl S, Lie JS, Bjorvatn B, Härmä M, Matre D. The association of sleepiness, insomnia, sleep disturbance and pain: a study amongst shift working nurses. Sleep Biol Rhythms. 2018;16:133-40.

4. Nowakowski S, Meers J, Heimbach E. Sleep and Women's Health. Sleep Med Res. 2013;4:1-22.

5. Komada Y, Ikeda Y, Sato M, Kami A, Masuda C, Shibata S. Subjective Sleep disturbance and psychological distress are associated with menstrual problems. J Womens Health Care. 2019;8:1-5.

Publisher's Note Springer Nature remains neutral with regard to jurisdictional claims in published maps and institutional affiliations. 